This item was submitted to Loughborough's Research Repository by the author.

Items in Figshare are protected by copyright, with all rights reserved, unless otherwise indicated.

\title{
Concentrator pointing control concept for fiber optic augmented solar thermal propulsion systems
}

\section{PLEASE CITE THE PUBLISHED VERSION}

http://dx.doi.org/10.2514/1.A33352

\section{PUBLISHER}

(c) American Institute of Aeronautics and Astronautics

\section{VERSION}

AM (Accepted Manuscript)

\section{PUBLISHER STATEMENT}

This work is made available according to the conditions of the Creative Commons Attribution-NonCommercialNoDerivatives 4.0 International (CC BY-NC-ND 4.0) licence. Full details of this licence are available at: https://creativecommons.org/licenses/by-nc-nd/4.0/

\section{LICENCE}

CC BY-NC-ND 4.0

\section{REPOSITORY RECORD}

Henshall, Paul, and Phil Palmer. 2019. "Concentrator Pointing Control Concept for Fiber Optic Augmented Solar Thermal Propulsion Systems”. figshare. https://hdl.handle.net/2134/19240. 


\title{
Concentrator Pointing Control Concept for
}

\section{Fiber Optic Augmented Solar Thermal}

\section{Propulsion Systems}

\author{
P. Henshall ${ }^{1}$ \\ Loughborough University, Loughborough, UK \\ P. Palmer ${ }^{2}$ \\ University of Surrey, Guildford, UK
}

\begin{abstract}
Nomenclature
$P \quad=\quad$ Power intercepted $(\mathrm{W})$

$x=$ Position/displacement along $\mathrm{x}$ axis (mm)

$y=$ Position/displacement along y axis $(\mathrm{mm})$
\end{abstract}

\section{Subscripts}

$1-7=$ Sensor, fiber optic reference

$A-D=$ Sensor, quadrant reference

\section{INTRODUCTION}

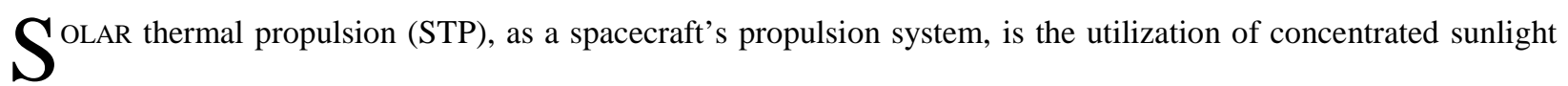
for the heating of a propellant to high temperatures via a heat exchanger. Sunlight concentration is achieved via

\footnotetext{
${ }^{1}$ Research Associate, Center for Renewable energy systems Technology, Loughborough University, LE11 3TU, UK (email: p.henshall@lboro.ac.uk)

${ }^{2}$ Professor, Surrey Space Centre. University of Surrey, GU2 7XH, UK. (email: p.palmer@surrey.ac.uk)
} 
an optical system, such as a series of lenses or mirrors. This concentrated sunlight impinges on a blackbody cavity receiver heating it to high temperatures. A propellant flows around the cavity receiver and heat is exchanged from the receiver to the propellant. The sunlight concentration system is orientated to point at the Sun, either via an onboard mechanism, or via the spacecraft attitude system. Solar thermal, mono-propellant, propulsion systems benefit from the utilization of a free energy source, they are capable of high energy conversion efficiencies (50 80\%) and have propulsive performances competitive with chemical bi-propellant systems (>350s) [1]. Given these apparent benefits, the motivation of this study was to investigate the feasibility of using a STP system on board a small-spacecraft. A small-spacecraft making use of the high propulsive performance offered by STP, could be considered for a wide variety of space mission applications.

In 2004, the use of fiber optics in a small-spacecraft STP system was proposed [1] [2]. With the application of fiber optics to a small-spacecraft STP system, a degree of flexibility with regard to system design and thrusting can be realized. For example, with fiber optics, the cavity receiver can be mechanically decoupled from the concentrator and placed anywhere on the spacecraft. The system mass can be significantly reduced as employing fiber optics allows multiple smaller concentrators, which can be independently oriented, to replace a single, larger, fixed concentrator (Fig. 1). Given that the mass of a (concentrator) mirror scales as the $4^{\text {th }}$ power of the diameter [3], these smaller mirrors are favored. However, this flexibility comes at a cost, as the energy travelling along the fiber is attenuated and reduces the end-to-end efficiency of the system. Previous tests of light transmission through fiber optics, at the Ben-Gurion University of the Negrev, have shown potential for transmission efficiencies as high as $80 \%$ [4]. It has been demonstrated that even with this decrease in efficiency the system still remains competitive with conventional propulsion systems [5]. However, despite this, there is the added complexity of a system that separately orientates each small solar concentrator, which ensures that solar energy from each concentrator is effectively coupled to the corresponding fiber optic. This must be achieved in a dynamic orbital environment with the individual pointing mechanisms subject to a variety of structural disturbances. Subsequently, a means of measuring the displacement between the solar image and the fiber optic tip on the concentrator focal plane is desirable, to provide feedback to concentrator pointing actuators and, thereby facilitating closed-loop pointing control. 


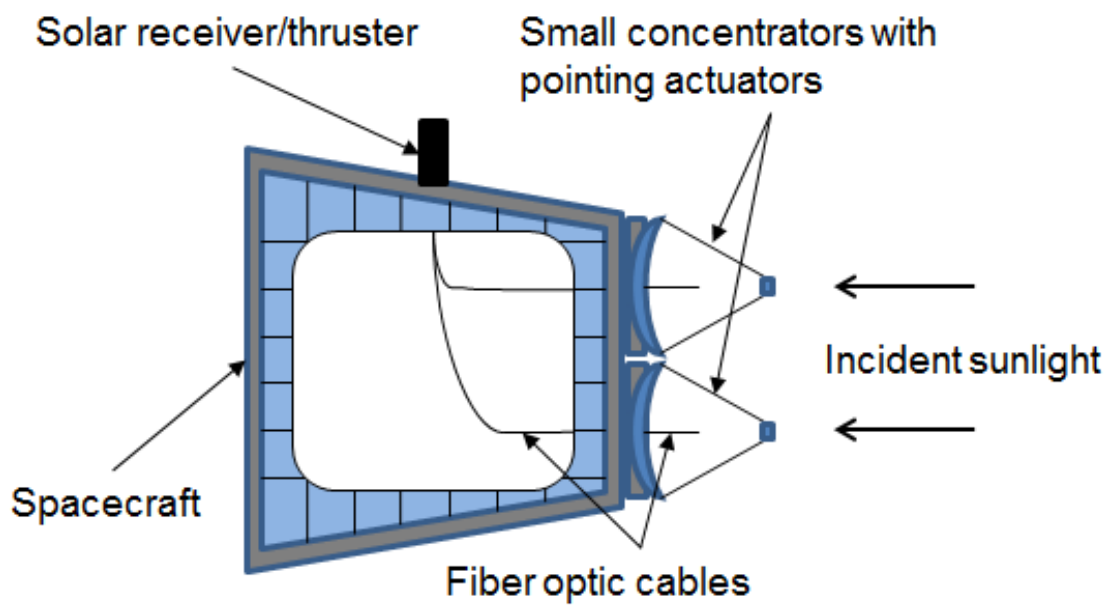

Fig. 1. Multiple small concentrator mirror concept for STP system on small spacecraft.

The current study describes a solution to this issue via a sensor strategy capable of measuring the displacement between the concentrator solar image and the fiber optic cable tip.

\section{Fiber Optic Pointing Sensor Concept ANd Modelling}

\section{A. Sensor Concept}

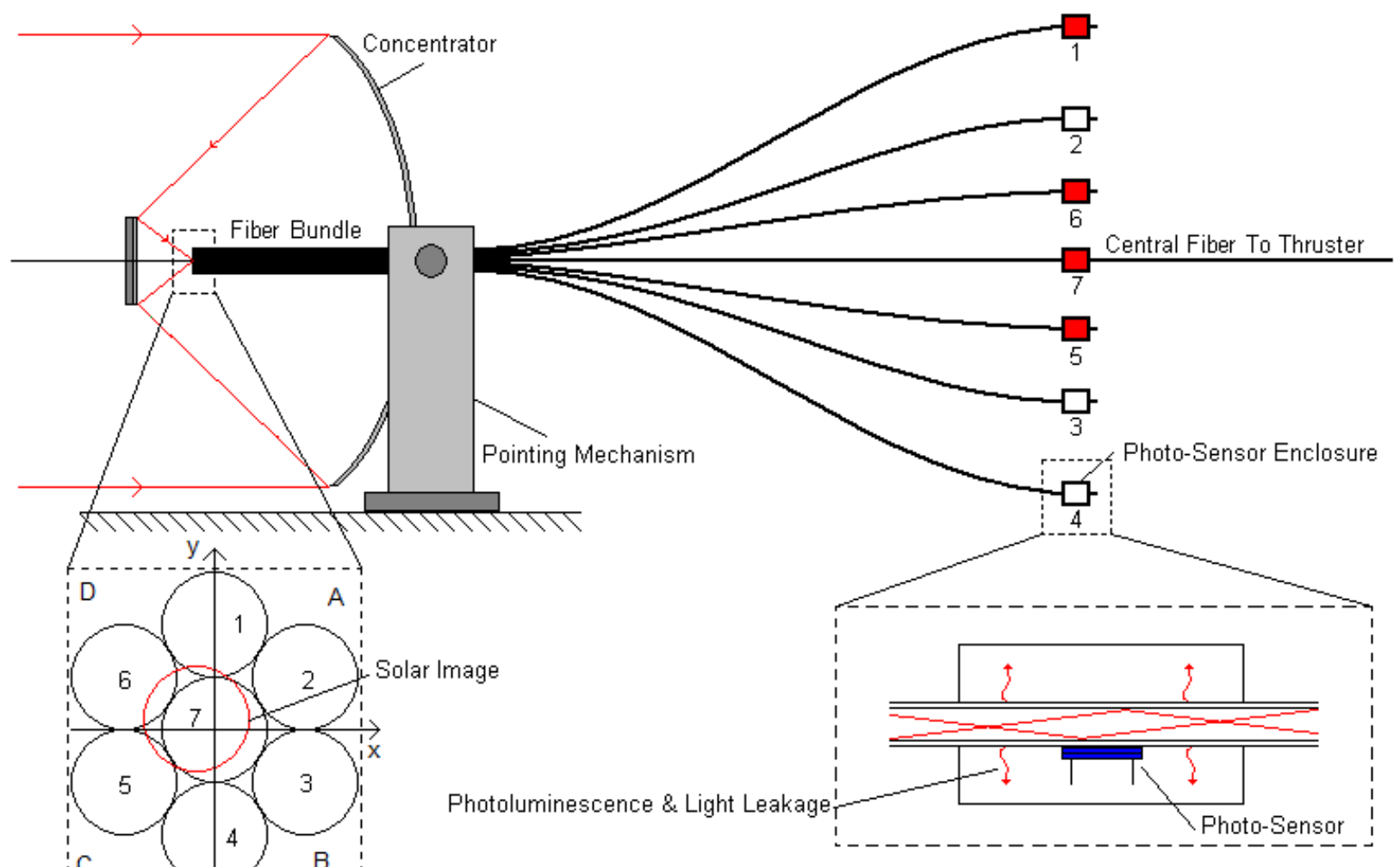

Fig. 2. Sensor Concept. 
The sensor concept is shown in Fig. 2 and consists of a bundle of seven fiber optics and an array of photodiodes. The solar image (referred to as the 'spot') at the focal point of a parabolic mirror concentrator impinges on the tip of the bundle, where the central fiber is primarily for transmission of concentrated solar energy and the surrounding fibers are primarily for providing spot position data. Each fiber passes through a small enclosure in which a photosensor (photodiode) is attached to its side, a certain distance from the concentrator end of the bundle. Photoluminescence and light leakage are documented transmission loss mechanisms in fiber optic cables [3] [6]. The photodiodes measure the photoluminescence and light leakage emitted by each fiber resulting from the high intensity light traversing the fiber. In this sense the fiber optics protect the photodiodes from being damaged via exposure to highly concentrated solar radiation. The signals from the photo-sensors are interpreted by a pointing determination algorithm to provide the position of the spot on the fiber bundle tip. This feedback information enables a closed-loop pointing control system, allowing the pointing actuation mechanism to track the Sun and compensate for spacecraft angular disturbances. In order to use this sensor concept, in the first instance, the concentrator must already be pointing at the Sun to an accuracy of $\pm 0.5^{\circ}$ such that the concentrator spot lies on the fiber bundle tip. This requires a solar concentrator pointing acquisition system to provide an initial pointing of the concentrator. This could be achieved by the host satellites attitude system combined with a Sun acquisition search algorithm. However, this study focuses on the fiber optic sensor only.

\section{B. Quadrant Pointing Algorithm Modelling}

An algorithm was devised to obtain estimates of Cartesian $(x, y)$ position of the center of the spot on the focal plane/fiber optic tip (Fig. 2). The $y$ direction is referred to as the "elevation" direction and the $x$ direction is known as the "azimuth" direction.

This algorithm splits the fiber bundle into quadrants A, B, C and D as shown in Fig. 3. This is analogous to laser pointing sensors in DVD players and the quad-cell Sun pointing sensor of the PICARD SODISM instrument [7]. Photodiodes measure the power intercepted by each fiber $\left(P_{1}-P_{7}\right)$, after which the power in each quadrant can be calculated as:

$$
P_{A}=P_{2}+\frac{1}{2} P_{1}+\frac{1}{4} P_{7}
$$




$$
\begin{aligned}
& P_{B}=P_{3}+\frac{1}{2} P_{4}+\frac{1}{4} P_{7} \\
& P_{C}=P_{5}+\frac{1}{2} P_{4}+\frac{1}{4} P_{7} \\
& P_{D}=P_{6}+\frac{1}{2} P_{1}+\frac{1}{4} P_{7}
\end{aligned}
$$

where it is necessary to apply the factors of $1 / 2$, to fiber 1 and 4 , and $1 / 4$, to fiber 7 , to correctly represent the powers in each quadrant. From equations 1 - 4 the estimation of the Cartesian location (x, y) of the spot on the fiber bundle tip can be estimated by comparing the powers of the bundle quadrants A, B, C and D as according to equations 5 and 6 .

$$
\begin{gathered}
x \propto \frac{\left(P_{A}+P_{B}\right)-\left(P_{C}+P_{D}\right)}{P_{A}+P_{B}+P_{C}+P_{D}} \\
y \propto \frac{\left(P_{A}+P_{D}\right)-\left(P_{B}+P_{C}\right)}{P_{A}+P_{B}+P_{C}+P_{D}}
\end{gathered}
$$

Using the fiber bundle as a quadrant sensor is more complicated than a normal quadrant sensor, as the gaps in between the fibers represent areas where the incident power cannot be measured. Also, due to the fiber geometry some fibers are located on the Cartesian axes which results in a coupling effect between the estimated $\mathrm{x}$ and $\mathrm{y}$ coordinates. To demonstrate this, the fiber bundle sensor was numerically simulated with a spot the same size as the fiber optics with tip diameter $(1 \mathrm{~mm})$ and with a square spot intensity profile. Fig. 3a plots the estimated $\mathrm{x}$ (or $\mathrm{y}$ ) position of the spot as it is moved along the $\mathrm{x}$ (or y) axis. This demonstrates that equations 5 and 6 can be used relatively accurately to describe the motion of the spot along the Cartesian coordinates frame axes, the best accuracy attainable via this method. The method accuracy decreases, however, as the spot is moved away from the $\mathrm{x}$ and $\mathrm{y}$ axes, due to a cross-coupling between the $\mathrm{x}$ and $\mathrm{y}$ coordinates. This coupling effect is shown graphically in Fig. 3 where Fig. $3 b$ is a contour plot of the estimated $\mathrm{x}$ position for varying actual y position and Fig. 3c is a contour plot of the estimated y position for varying actual x position. It is clear from these figures that the methods accuracy decreases with distance from the coordinate origin. Despite this, the method is still able to provide an estimate of the spot position and can accurately locate the center of the fiber optic bundle. 


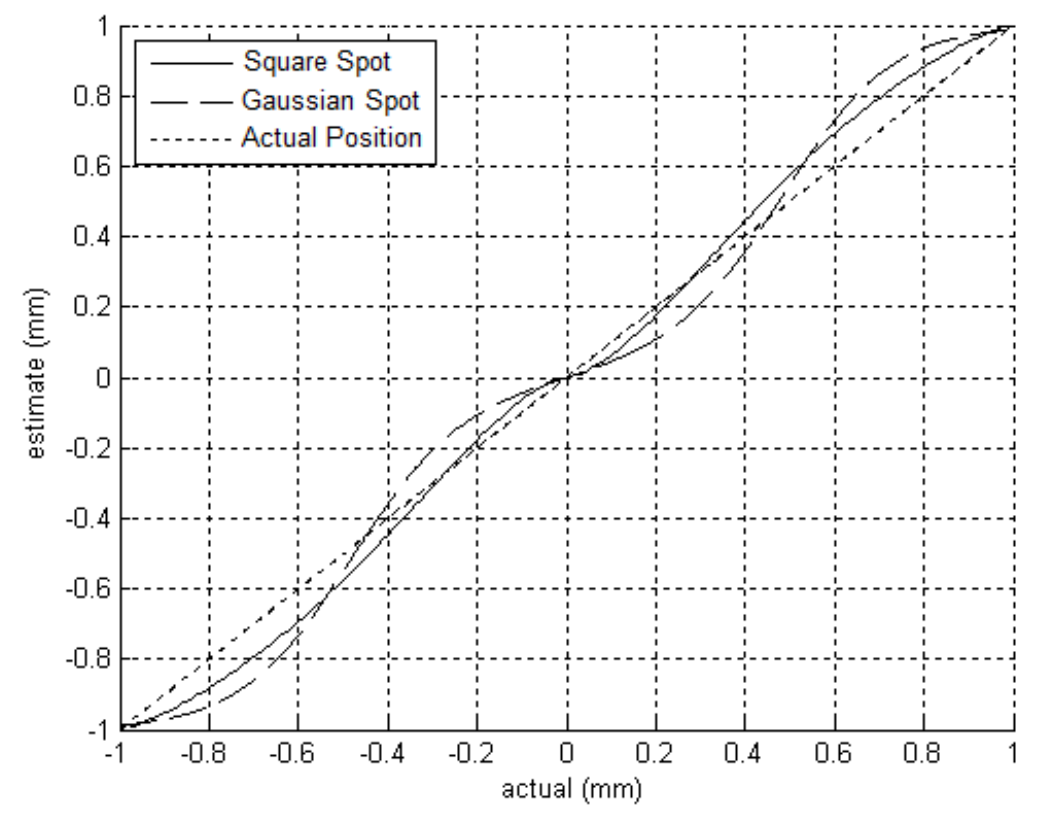

(a)

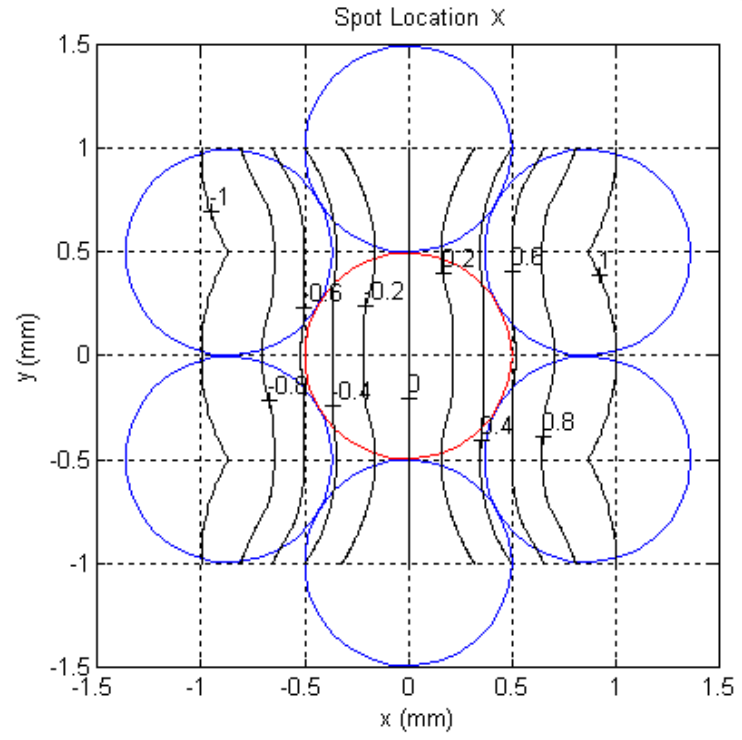

(b)

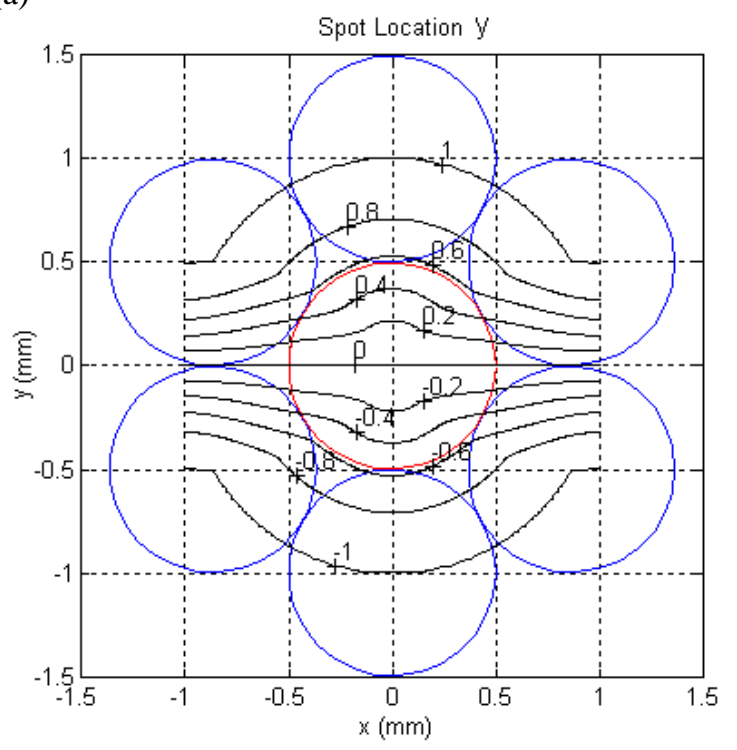

(c)

Fig. 3. Quadrant algorithm (a): $x$ (or $y$ ) axis coordinate error for different spot intensity distributions, (b): $x$ coordinate contours, (c): y coordinate contours.

A further influence on algorithm estimation accuracy is the variation of spot intensity as a function of distance from the center of the spot. The concentrators manufactured for experimental testing of the sensor were aluminum, diamond turned to an RMS surface error of $\sim 0.1 \mu \mathrm{m}$ (concentrator properties: aperture diameter: $105 \mathrm{~mm}$, rim angle: 40, F-number: 0.687, focal length: 72.1mm, concentration ratio: 11000 and Strehl ratio: 0.8 ). Ray tracing analysis 
of these parabolic concentrator mirrors indicated that the intensity moving towards the edge of the spot would tailoff in a Gaussian manner regardless of the model used to reflect the solar disk intensity [4]. Experimental testing with a solar simulator providing collimated light of the correct intensity, suggested that in this case the spot intensity profile of the $105 \mathrm{~mm}$ concentrator spot was Gaussian like in the $\mathrm{x}$ direction, with a standard deviation of $0.25 \mathrm{~mm}$ and an effective spot diameter of $1.5 \mathrm{~mm}$. This effective spot diameter is slightly bigger than expected; however, this is likely due to non-perfect collimation of the beam. Subsequent modeling of the sensor with a similar Gaussian spot intensity distribution is also shown in Fig. 3a. The simulated Gaussian spot intensity distribution results in an increased curvature of the error curve and a subsequent decrease in sensor accuracy.

\section{SENSOR EXPERIMENTAL TESTING}

\section{A. Investigation of fiber optic photoluminescence}

The aim of this investigation was to assess the behavior of the photoluminescence exhibited by a Polymicro [8], FLU fiber optic cable, with a 1mm tip diameter - the fiber optic selected for this application - when transmitting concentrated sunlight. In this investigation the main focus of the experiment was the variation in the photoluminescence intensity and the resulting variation in photo-sensor response, rather than any spectral variation. The photo-sensor selected to be suitable for this application was the OPT101P photodiode, a product of Burr-Brown [9]. Experimentally, it was found that these photodiodes exhibit a linear response in relation to the photoluminescence resulting from concentrated solar energy being transmitted through the fiber optic cable.

\section{B. Sensor testing}

A bundle of seven fibers was constructed using high temperature epoxy resin, and an array of seven photodiode sensors, powered in a series circuit, was manufactured. An XPC-Target data acquisition system, which operates within Simulink, was used to monitor the seven photodiodes and log their output voltage responses (Fig. 4). 


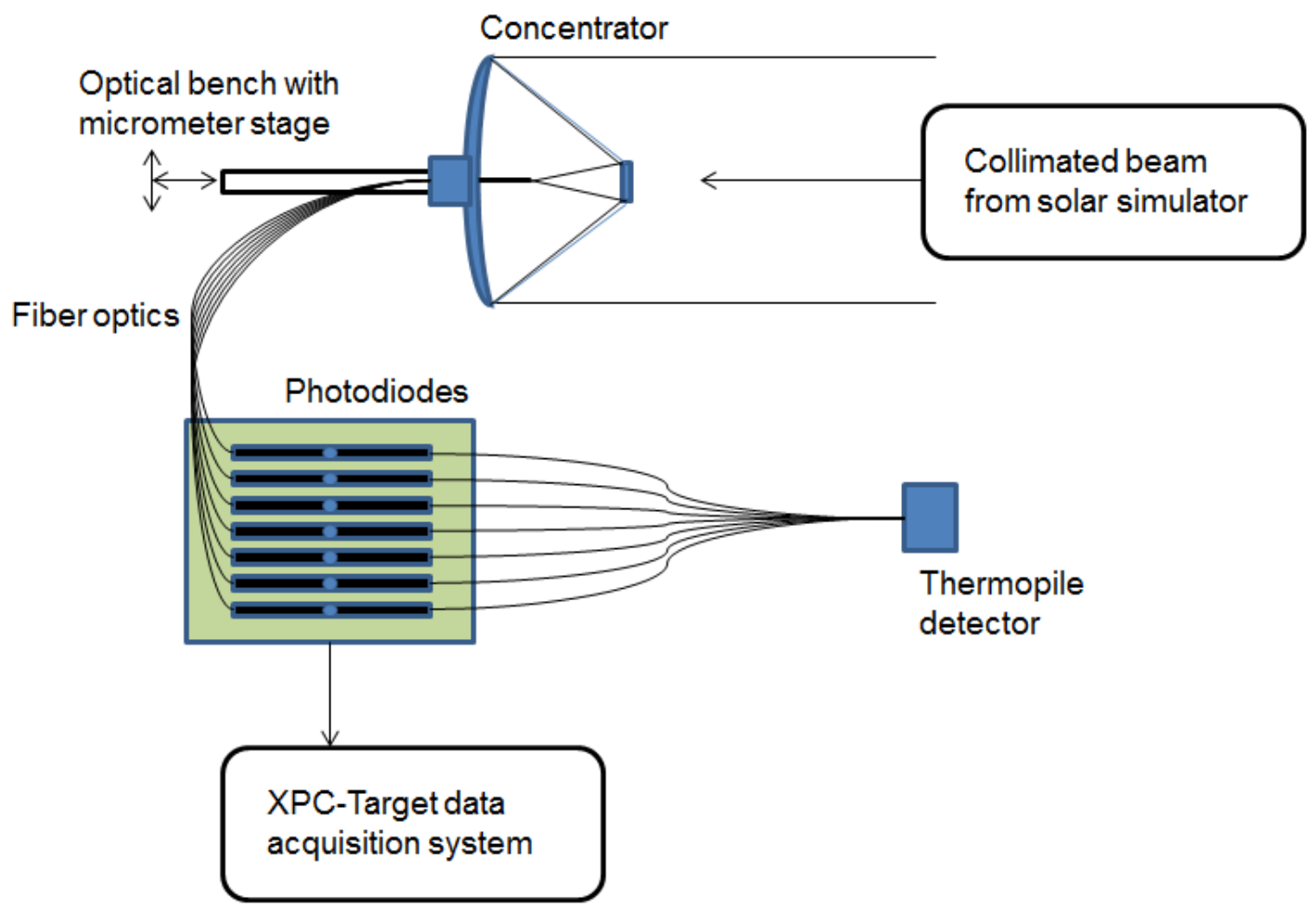

Fig. 4. Experimental setup

The experimental setup is depicted in Fig. 4. The 105mm parabolic mirror concentrator (with a secondary mirror in a Cassegrain arrangement) was located on an optical bench and integrated with a micrometer stage, used to record and adjust actual spot location. The concentrator was aligned with a collimated beam, originating from a solar simulator. A thermopile detector was used to monitor total power transmitted along the fiber bundle as well as individual fiber optics.

A preliminary sensor test was formulated to determine if individual fibers in the bundle were responding in the correct manner, relative to one another. The tests involved quickly moving the spot over the fiber tip from top to bottom along the y axis (see Fig. 2) and ensuring photodiode responses were consistent with expected sensor behavior. Using the logged photodiode response test data, the estimated spot position over the course of the test was calculated via the quadrant algorithm (see Fig. 5). In Fig. 5 the error signals are clean and provide good nonilluminated responses. However, it should be noted here that the azimuth error signal may either indicate that the fiber bundle tip is slightly tilted to one side, or that a small amount of cross-coupling is occurring. 


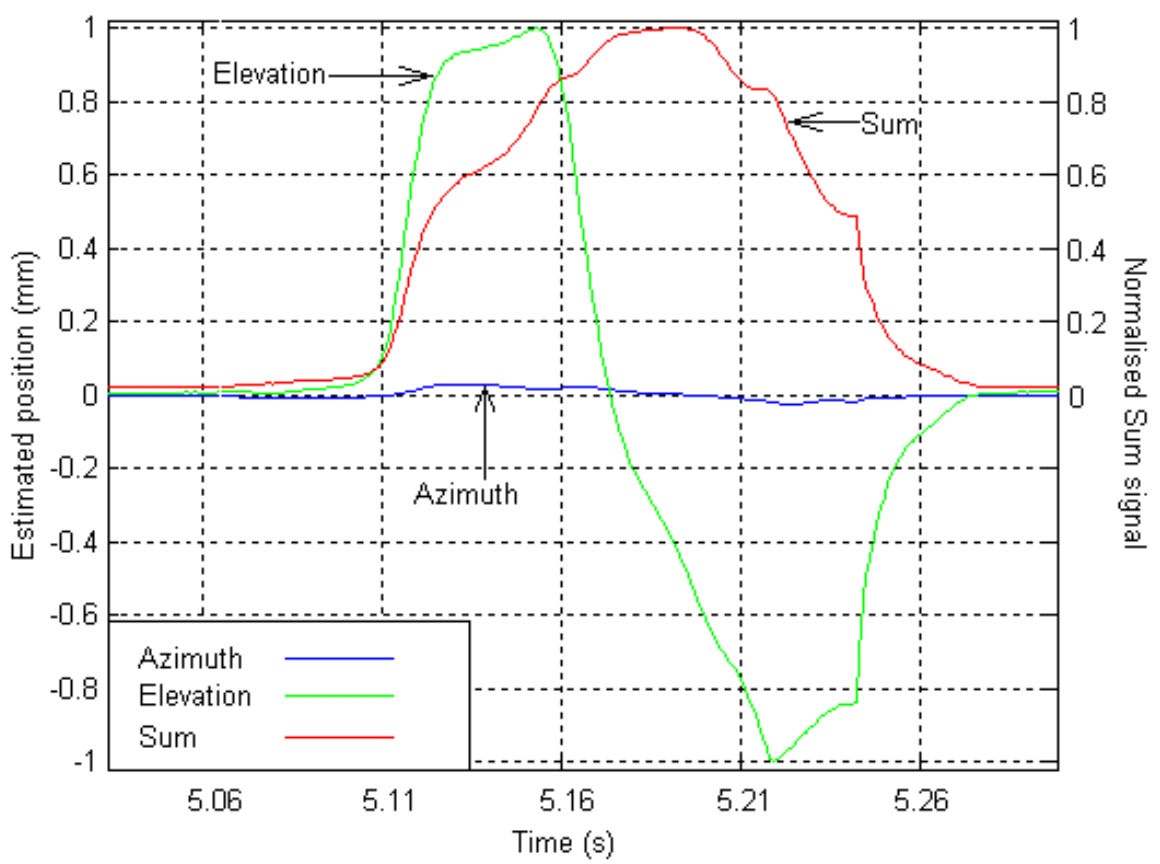

Fig. 5. Algorithm calculated position for sensor photodiode response test $( \pm 0.04 \mathrm{~mm})$.

A further test was performed to access the relation between the estimated and actual spot position. This test was performed via manually adjusting the location of the spot via a micrometer stage while logging the photodiode response data, which was then processed via the quadrant algorithm to provide $\mathrm{x}$ and $\mathrm{y}$ position estimates.

In Fig. $6 a$ and b, the relations between actual spot position and estimated spot position can be seen for both the azimuth and elevation directions. Both relations exhibit responses with similar features to the simulated relations shown in Fig. 3a. For example, the plots exhibit a curvature pattern consistent with that seen in Fig. 3a. However, the relations shown in Fig. 6a and b do occasionally deviate from those in Fig. 3a. In Fig. 6a the sensor over predicts the azimuth actual position of the spot near the center of the bundle, whereas modelled results suggest the sensor would under predict in this region; as is seen in Fig. 6b. Additionally, in Fig. 6b, the sensor's behavior is nonsymmetrical. Such deviations could be attributable to a slightly larger concentrator spot size, or to the spot intensity profile being centrally flatter and subsequently not precisely Gaussian or symmetrical. Other sources of error may include error in fiber response calibration, fiber optic cladding effects and the non-perfect elimination of back ground light in the fiber optic sensor box. However, these tests demonstrated that the new fiber optic bundle sensor was functioning satisfactorily, was relatively consistent with predicted behavior and was capable of accurately locating the center of the fiber optic bundle. 


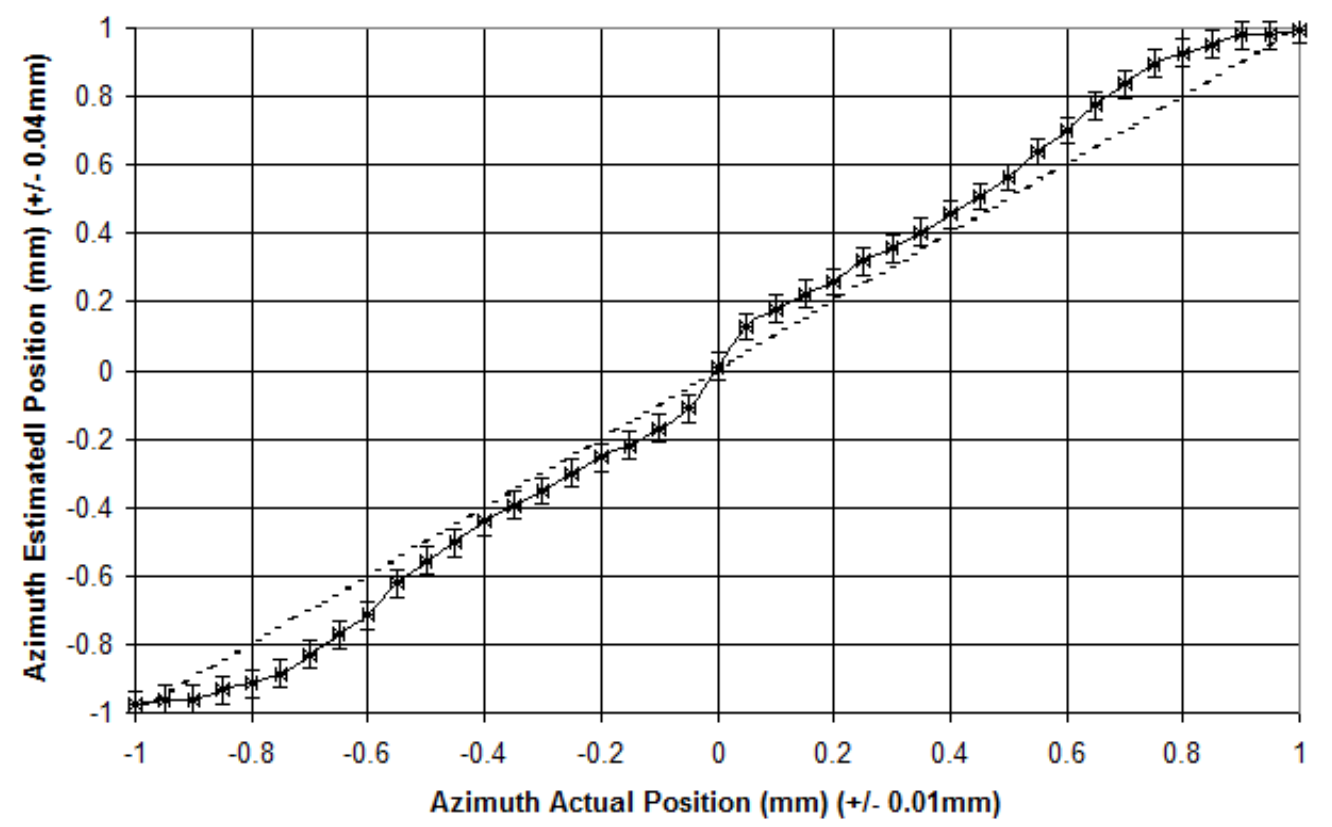

(a)

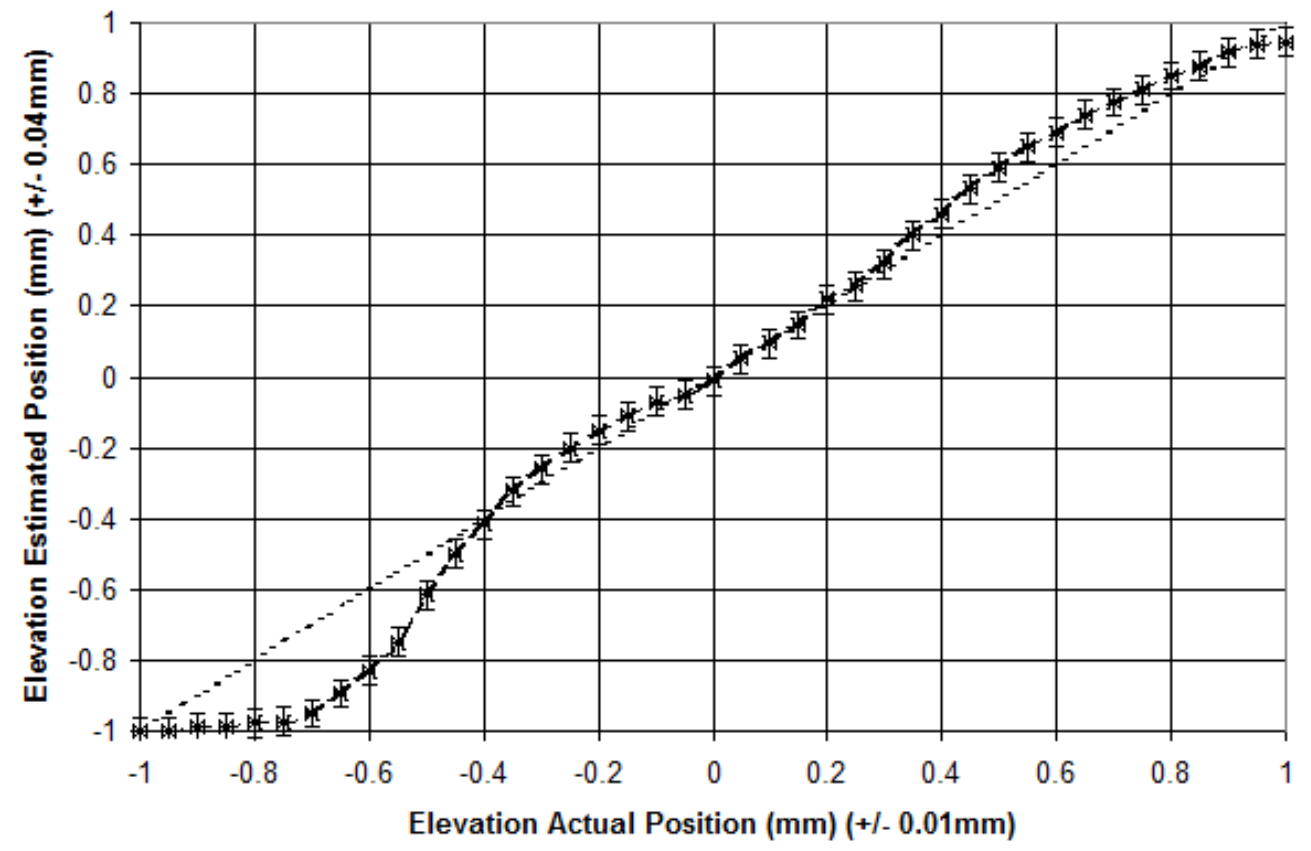

(b)

Fig. 6. (a) Azimuth, measured position vs. actual position. (b) Elevation, measured position vs. actual position.

\section{CONCLUSION}

In this paper a novel fiber optic sensor concept was introduced, capable of providing pointing telemetry for a solar concentrator pointing control system. A sensor algorithm was discussed and efforts to manufacture a working fiber optic sensor described. The quadrant pointing algorithm demonstrated reasonable robustness to channel cross 
coupling effects and reasonable pointing error determination; being able to accurately locate the center of the fiber bundle in modelled and experimental cases. Further work includes improving the sensor accuracy and consistency over the area of the fiber bundle tip and integrating the sensor with control and mechanical activation systems. It is anticipated that the combination of these technologies would be a viable means of providing closed loop control to solar concentrator pointing mechanisms for a small spacecraft STP system.

\section{ACKNOWLEDEMENTS}

Alexandre Pechev. This work was supported by the European Office of Aerospace Research and Development (EOARD).

\section{REFERENCES}

[1] Kennedy, F.G., Henshall, P. R., and Gibbon, D., "Preparing for Flight: The Surrey Space Centre’s Microscale Solar Thermal Propulsion Experiment (MSPEX)," 4th International Space Propulsion Conference, 2004.

[2] Nakamura, T., Sullivan, D., McClanahan, J. A., Shoji, J. M., Partch, R., and Quinn, S., "Solar Thermal Propulsion for Small Spacecraft,” AIAA Joint Propulsion Conference, Fort Lauderdale, Florida, 2004.

[3] Henshall, P.R., "Applications of Fibre Optics in Solar Thermal Propulsion Systems", PhD Thesis, University of Surrey, 2007.

[4] Feuermann, D., and Gordon, J. M., "Solar Fibre-Optic Mini-Dishes: A New Approach To The Efficient Collection Of Sunlight,” Solar Energy, vol. 65, no. 3, 1999.

[5] Kennedy, F. G., "Solar Thermal Propulsion for Microsatellite Manoeuvring", PhD Thesis, University of Surrey, 2004.

[6] Sakurai, Y., and Nagasawa, K., "Excitation energy dependence of the photoluminescence band at 2.7 and $4.3 \mathrm{eV}$ in silica glass at low temperature," Journal of Non-Crystalline Solids, 2001.

[7] Dame, L., Brun, J., Cugnet, D., Derrin, M., Leyroy, C., Meftah, M., Meissonnier M., and Portuenuve, J., “A Solar Diameter Metrology Measurement: The PICARD Microsatellite Program,” CNRS internal report.

[8] Polymicro, "Polymicro Technologies Optical Fibers," Polymicro, [Online]. Available: http://www.polymicro.com/molex/products/family?key=polymicro_optical_fibers\&channel=products\&chanNa me=family\&pageTitle=Introduction. [Accessed 122 2015].

[9] Farnell, "Monolithic photodiode and single-supply transimpedance amplifier," [Online]. Available: www.farnell.com/datasheets/53029.pdf. [Accessed May 2005]. 